\title{
Análisis de las redes sociales en la vejez a través de la entrevista Manheim
}

\author{
Juan Carlos Meléndez-Moral, D en Psic,, ${ }^{(1)}$ José Manuel Tomás-Miguel, D en Psic, (2) Esperanza Navarro-Pardo, D en Psic. ${ }^{(1)}$
}

Meléndez-Moral JC,Tomás-Miguel JM, Navarro-Pardo E. Análisis de las redes sociales en la vejez a través de la entrevista Manheim. Salud Publica Mex 2007;49:408-4I4.

\begin{abstract}
Resumen
Objetivo. Reconocer quiénes son las figuras suministradoras de apoyo, psicológico e instrumental, en la vejez, así como precisar si existen diferencias en función de la edad y el sexo. Material y métodos. Se utilizó la entrevista Manheim de apoyo social y se recogieron las opiniones de $10 \mathrm{I}$ personas mayores ( $>65$ años) de la provincia deValencia (España), entre enero y mayo de 2006. Resultados. Se han encontrado diferencias estadísticamente significativas en el apoyo psicológico y el instrumental y tanto en relación con la edad como con el sexo del entrevistado. Conclusiones. Respecto del género, los datos muestran que los hombres reciben más apoyo de sus parejas, mientras que las mujeres tienden a priorizar a los hijos como suministradores de apoyo.Acerca de la edad, las parejas son más importantes en la primera de las franjas de edad consideradas (65-75 años) y a medida que la edad aumenta los hijos las reemplazan.
\end{abstract}

Palabras clave: apoyo psicológico; apoyo instrumental; envejecimiento; edad; sexo; Valencia
Meléndez-Moral JC, Tomás-Miguel JM, Navarro-Pardo E. Analyses of the social networks for the elderly

through the Manheim interview.

Salud Publica Mex 2007;49:408-4I4.

\section{Abstract}

Objective.To analyse who are the people giving psychological and instrumental support to the elderly, as well as potential differences among these caregivers according to age and/or sex of the elderly. Material and Methods. Manheim interview for social support was used to gather the opinions of $10 \mathrm{I}$ elder people (>65 years) living in Valencia (Spain), from January to May, 2006. Results. Statistically significant differences were found on both psychological and instrumental support depending on age and sex of the interviewed elderly. Conclusions. With respect to sex differences old men seek for support mainly from their partners, while son and daughters are the main source of support for old women. With respect to age, there are changes in the figures giving support as age increases. Partners are the main figure to give support within the 65-75 years, but its importance decreases with age, while sons and daughters became more important as age of the elderly increases.

Key words: psychological support; instrumental support; ageing; age; sex; Valencia
A 1 describir el concepto de apoyo, una de las definiciones más citadas lo refiere como el conjunto de provisiones expresivas o instrumentales (percibidas o recibidas) proporcionadas por comunidades, redes sociales y personas de confianza, sin soslayar que estas provisiones se pueden producir en situaciones cotidianas o de crisis. ${ }^{1}$ Por otra parte, y en adición a esta definición, también parece importante distinguir

(I) Departamento de Psicología Evolutiva y de la Educación, Universitat de Valencia.Valencia, España.

(2) Departamento de Metodología de las Ciencias del Comportamiento, Universitat de Valencia.Valencia, España.

Fecha de recibido: 18 de abril de 2007 - Fecha de aceptado: I I de julio de 2007 Solicitud de sobretiros: Dr. Juan Carlos Meléndez-Moral. Departamento de Psicología Evolutiva y de la Educación. Facultad de Psicología, Universidad de Valencia. Av. Blasco Ibáñez 2I, 460IOValencia, España.

Correo electrónico: melendez@uv.es 
entre dos perspectivas teóricas que existen en el estudio de este concepto: la estructural y la funcional, ${ }^{2}$ que se recogen en este trabajo.

De estas perspectivas, la estructural se interesa por el análisis de las condiciones objetivas del proceso de apoyo y la estructura de las relaciones; además, establece su centro de análisis en las redes sociales, entendidas como las características estructurales observadas en las interacciones que se mantienen con otras personas.

Por otro lado, la perspectiva funcional analiza la función que esas características cumplen para el sujeto, que se clasifican de manera genérica en instrumentales y expresivas. Por consiguiente, se introduce una diferencia entre los fines y los medios de la acción, de tal modo que la función instrumental hace referencia a los actos que la persona emprende para lograr unos fines, que difieren de los medios para conseguirlos, mientras que la función expresiva alude a las acciones que el individuo realiza y cuyo fin son ellas mismas.

Sin embargo, el apoyo, así como la red social, no es constante y experimenta transformaciones a lo largo del desarrollo y modificaciones, no sólo cualitativas sino cuantitativas. Al analizar el ciclo vital se puede observar cómo en los primeros años de vida la red social es prácticamente inexistente y está centrada en las conductas de apego; tales sentimientos se desarrollan a medida que el niño incrementa la confianza en sus cuidadores principales, así como en las personas importantes de su entorno.

Se debe tener en cuenta que el apego es un vínculo afectivo duradero de carácter singular, que se desarrolla, prospera y consolida por medio de la relación activa y recíproca de las partes participantes, ${ }^{3}$ y que estas relaciones condicionan su desarrollo posterior, ya que constituyen la base para las futuras relaciones sociales. ${ }^{4}$

Más adelante, y ya en las primeras etapas de la infancia, la red social se incrementa y da paso a la aparición de otras relaciones sociales significativas, si bien hay que destacar que la posibilidad que tienen los niños de extender su red social depende en buena medida de las decisiones que toman sus padres. Además, dichas interacciones que no poseen carácter de continuidad temporal, se forman y disuelven con cierta facilidad y, por lo tanto, los amigos se perciben como compañeros provisionales de juego.

Con base en esta evolución, es en los años escolares cuando el niño comienza a desarrollar nuevas capacidades de autonomía que emergen a partir del ingreso en el mundo social de la escuela. ${ }^{5}$ En este nuevo contexto, el niño expande las redes interpersonales y progresivamente concede mayor importancia a las relaciones con los iguales. En dicha etapa, la amistad se define por la cooperación y la ayuda recíproca y los amigos se conciben como personas que se ayudan en una relación de confianza mutua.

Con la llegada de la adolescencia, la red social vuelve a sufrir una nueva transformación, al ampliarse y convertirse en una fuente de estima y aceptación por parte de otras relaciones distintas del círculo familiar. La evolución de esta red es la consecuencia de un proceso continuo de transacciones y selección mutua; los atributos personales, así como los intereses y necesidades, son los ejes en función de los cuales se genera la pertenencia a los diferentes grupos.

De nuevo, la transición hacia la juventud y edad adulta implica modificaciones importantes en los planos estructural y funcional y supone el aprendizaje de nuevas tareas y roles. En consecuencia, el inicio de relaciones íntimas y estables exige la aceptación de las diferencias personales y la existencia de distintas expectativas. Además, aparece la creación de redes que rebasan el ámbito de la pareja.

En esta etapa, también el mundo del trabajo se convierte en una fuente de apoyo, tanto en las relaciones profesionales como en las personales. Asimismo, la pareja se convierte a menudo en la fuente de apoyo más importante y da paso a nuevas transformaciones derivadas de esta relación; más aún, en el caso de tener hijos lleva a nuevas orientaciones centradas en los contactos con la familia y los amigos.

Por último, con la llegada de la jubilación, la edad, como característica evolutiva, recobra fuerza como influencia normativa y vuelve a ser un importante determinante del cambio. En este sentido, la red social en edades avanzadas muestra una serie de cambios típicos, como la finalización de la crianza de los hijos, el abandono de las relaciones procedentes del ámbito laboral y la disminución del grupo de parientes e iguales por efecto de la mortalidad. De esta manera, y aunque durante gran parte del ciclo vital la red de apoyo se ha extendido, a partir de los 70 años sufre al parecer un descenso. ${ }^{6}$

Este dato podría ser indicativo de la aparición de problemas generados por la falta de apoyo, pero la reducción de los contactos sociales y la progresiva desaparición de las relaciones íntimas las asumen las personas mayores debido a la tendencia a aplicar conductas de tipo acomodativo como forma de adaptarse a las nuevas situaciones. ${ }^{7}$ Así, y mediante este tipo de estrategias, logran generar un ajuste de las preferencias y las metas que, incluso si no es intencional, hace que el sujeto se adapte de manera adecuada a la nueva situación y atenúe la percepción de las restricciones situacionales o unos recursos personales insuficientes para conseguir objetivos antes valorados. El envejecimiento, implica 
una reducción de los recursos personales de acción y las opciones evolutivas disponibles, lo cual tiende a favorecer la acomodación, en comparación con la asimilación, como forma de adaptación y optimización de los recursos.

En este sentido, se debe tener en cuenta también que la reducción del tiempo que queda para conseguir las metas es un elemento fundamental en el reajuste, ya que modifica las expectativas futuras y lleva a establecer objetivos a más corto plazo y más modestos. Por lo tanto, los mayores intentan primordialmente conservar las cosas buenas, todavía disponibles y posibles en su presente; ${ }^{8}$ en tal sentido, envejecer, a través de estos movimientos de acomodación, deja de convertirse en una cuestión de ganar y alcanzar nuevos estados para pasar a ser en esencia una cuestión de no perder lo que ya se tiene y mantenerse ante un incierto futuro. Asimismo, debe pensarse que en muchos casos los mayores ya disfrutan de las metas conseguidas a través de los años, con lo que mantenerlas se convierte en algo con pleno sentido.

Por último, hay que resaltar dos aspectos fundamentales, uno referido a la procedencia del apoyo y otro a la función de éste.

En relación con el primer punto, y en el contexto español, $71.9 \%$ del apoyo recibido por parte de los ancianos procede del apoyo informal: la familia cercana (cónyuge e hijos) es la mayor proveedora (63.8\%), seguida de parientes, amigos y vecinos, que representan $8.1 \%$ del total. ${ }^{9}$ En esta misma línea de resultados, y en el contexto de México, según Montes de Oca, ${ }^{10}$ la encuesta ENSE-94 muestra que $74.6 \%$ del apoyo recibido lo conceden el cónyuge (17.65\%) y los hijos (56.95\%); destacan también de entre las demás figuras de apoyo, los yernos y nueras con $5.85 \%$ y los nietos con 5.3 por ciento. Estos resultados son similares a los obtenidos mediante la encuesta ENASEM-2001,11 realizada también en México.

En cuanto a las funciones del apoyo en el envejecimiento, está demostrado que éste tiene efectos beneficiosos al enfrentar situaciones estresantes que requieren adaptación y preserva al sujeto de los posibles cambios negativos en el estado de salud, física y mental. ${ }^{12}$ En este sentido, las relaciones de apoyo fomentan, desarrollan y ayudan a mantener la percepción y sentido de control de un individuo, ${ }^{13}$ lo cual puede minimizar la pérdida de control primario que se produce con la edad $^{14}$ y ayudar a generar conductas derivadas del control secundario. De igual forma, el apoyo social es un factor importante dentro del concepto de bienestar y el envejecimiento satisfactorio y óptimo, ${ }^{15}$ y un facilitador de las conductas de promoción de la salud; esto atenúa el riesgo de mortalidad e internamiento.
En consecuencia, este trabajo tiene como objetivo reconocer las principales figuras de apoyo psicológico e instrumental en el envejecimiento en función de la edad y el sexo.

\section{Material y métodos}

La muestra analizada es de 101 personas que viven en la provincia de Valencia (España), mayores de 65 años y jubilados; las entrevistas se efectuaron entre enero y mayo de 2006. El muestreo se realizó mediante encuestadores que debían cumplir cuotas respecto del sexo, la mitad varones y la mitad mujeres. La media de la edad fue de 73.48, la mediana de 73 años y la desviación típica de 6.62. El $49.5 \%$ correspondía a hombres y $50.5 \%$ a mujeres. Una proporción de $63 \%$ se integraba con casados, $1.2 \%$ con solteros y $35.8 \%$ con viudos.

Este estudio se sometió al Comité de Ética de la Facultad de Psicología de la Universidad de Valencia y el trabajo se llevó a cabo con el consentimiento informado de los participantes.

\section{Medidas}

El instrumento utilizado para este trabajo es una adaptación ${ }^{16}$ de la entrevista Manheim de Apoyo Social. ${ }^{17}$

El marco conceptual en el que ésta se basa tiene en cuenta la diferenciación de los tipos de apoyo y sus funciones, sus fuentes y los diferentes focos de evaluación, de tal modo que aporta una perspectiva mixta en la que se analizan los elementos funcionales y los estructurales.

La versión española ${ }^{18}$ se compone de 22 preguntas, abiertas y de tipo Likert, divididas en tres partes. La primera explora los tipos que apoyo definidos desde la perspectiva funcional y analiza a las personas que proporcionan apoyo psicológico o instrumental en situaciones cotidianas y de crisis. La segunda parte recoge aspectos de la perspectiva estructural, suministra información descriptiva y evaluativa acerca de las personas mencionadas en la primera parte y hace posible definir de forma más detallada las fuentes de apoyo y el foco de evaluación, que son elementos descriptivos (frecuencia de contacto, tamaño de la red de apoyo, etc.). En la tercera parte se evalúa la actitud del entrevistado hacia la búsqueda y recepción del apoyo social.

Para los intereses de este trabajo se utilizó la primera parte de la entrevista, con las medidas de apoyo psicológico e instrumental obtenidas de las diferentes preguntas mediante adición del apoyo cotidiano y en crisis. Además, y dentro de la exploración que la entrevista hace respecto de las personas que potencialmente 
ofrecen apoyo, se seleccionaron las primeras elecciones, las más relevantes. Para ello se procedió, en primer lugar, a analizar las respuestas acerca de la procedencia del apoyo que reciben los entrevistados; se categorizaron los siguientes niveles: cónyuge o pareja, hijos, nietos, otros familiares y amigos. Con posterioridad se realizó un recuento del número de veces que éstos se identificaban como figuras relevantes tanto para el apoyo psicológico como para el instrumental.

\section{Análisis estadísticos}

Se analizaron las relaciones entre las variables edad y sexo y las elecciones de las figuras de apoyo; mediante análisis factorial de correspondencias simple para analizar las distancias entre las categorías de las variables nominales a analizar, de formas descriptiva y exploratoria, y además se emplearon la prueba de la ji cuadrada y las medidas de $\mathrm{V}$ de Cramer para evaluar el tamaño del efecto. ${ }^{19}$ Los análisis factoriales de correspondencias se realizaron mediante distancias de la ji cuadrada, centrado de filas y columnas y normalización simétrica. Todos los análisis estadísticos se llevaron a cabo con el paquete estadístico SPSS 14.

\section{Resultados}

Como objetivo del trabajo se planteó una cuestión cualitativa importante: ¿de quién procede el apoyo, tanto psicológico como instrumental? Además, otra finalidad fue determinar si había diferencias en función de la edad y el género.

Así pues, se analizó en primer lugar la relación entre el sexo y la figura que ofrece apoyo psicológico; se observó así una relación significativa entre ambas variables $\left(\chi_{4}^{2}=47.795, p<0.001, \mathrm{~V}=0.275\right)$. En el cuadro I se presentan los porcentajes obtenidos.

Por su parte, las relaciones entre el sexo y la figura que suministra apoyo instrumental también resultaron estadísticamente significativas $\left(\chi_{4}^{2}=25.624, p<0.001\right.$, $\mathrm{V}=0.239$ ). En el cuadro II se presentan los porcentajes obtenidos.

De la misma forma, se analizaron las relaciones de la figura que ofrece apoyo con la edad de los entrevistados. En relación con el apoyo psicológico, una primera prueba de la ji cuadrada proporcionó resultados estadísticamente significativos $\left(\chi_{8}^{2}=54.79, p<0.001\right.$, $\mathrm{V}=0.208$ ). En este caso, y en virtud del mayor número de categorías de la variable edad, se optó por un análisis factorial de correspondencias simple, con el objeto de entender las proximidades entre las categorías de ambas variables. Este análisis factorial de correspondencias mostró un resultado de dos dimensiones, de las cuales

\section{Cuadro I}

Porcentajes en función del SEXo de La figura QUE OFRECE APOYO PSICOLÓgico.VAlencia, España

\begin{tabular}{llllllll} 
& \multicolumn{6}{c}{ ¿Quién da apoyo psicológico? } & \\
\cline { 2 - 6 } & Amigos & Cónyuge & Hijos & Nietos & Otros familiares & Total \\
Hombres & $17.4 \%$ & $42.2 \%$ & $24.5 \%$ & $5.3 \%$ & $10.6 \%$ & $100 \%$ \\
\hline Mujeres & $19 \%$ & $19 \%$ & $45.3 \%$ & $5.5 \%$ & $11.3 \%$ & $100 \%$
\end{tabular}

\section{Cuadro II \\ Porcentajes en función del sexo de la figura QUE OfReCE APOYO INSTRUMENTAL.VAlencia, España}

\begin{tabular}{lccccccc} 
& \multicolumn{6}{c}{ ¿Quién da apoyo instrumental? } & \\
\cline { 2 - 6 } & Amigos & Cónyuge & Hijos & Nietos & Otros familiares & Total \\
Hombres & $9.9 \%$ & $26 \%$ & $41.3 \%$ & $3.6 \%$ & $19.3 \%$ & $100 \%$ \\
\hline Mujeres & $3.5 \%$ & $15.9 \%$ & $63.3 \%$ & $1.3 \%$ & $15.9 \%$ & $100 \%$
\end{tabular}

la primera explica $75.5 \%$ de la inercia y se definió por la oposición entre la muestra de menor y la de mayor edad, con contribuciones absolutas de $-0.668,0.431$ y 0.340 , respectivamente. Por cuanto a las categorías de la figura que provee el apoyo psicológico, la dimensión primera se definió por la oposición entre el cónyuge (-0.597) y los hijos (0.465) y nietos (1.07). En relación con la segunda dimensión, mucho menos relevante pues representa $24.5 \%$ de la inercia, quedó definida en las categorías de edad por la oposición entre los de mayor edad ( 75 o más, contribución de 0.45$)$ y las otras edades, en particular los de 70 a 74 (-0.502). Por su parte, las categorías de figura que ofrece apoyo definieron esta dimensión por la oposición de hijos (0.386) frente a nietos (-1.139). Las cercanías entre categorías se pueden observar mediante el diagrama dual de la figura 1.

A continuación se realizó otra prueba de la ji cuadrada, esta vez para relacionar la edad y la figura que ofrece apoyo instrumental y también se identificó una relación estadísticamente significativa $\left(\chi_{8}^{2}=38.578\right.$, $p<0.001, V=0.207)$. Además, se realizó el análisis factorial de correspondencias y se observaron los resultados de las dos dimensiones obtenidas en la figura 2. De esa forma, la primera dimensión, que fue sin duda la de mayor importancia, acumuló $96.8 \%$ de la varianza. De nueva cuenta, esta dimensión se definió por las edades, de menor (-0.738) a mayor (0.47); en cuanto a las figuras que ofrecían apoyo instrumental, en un polo de la di- 


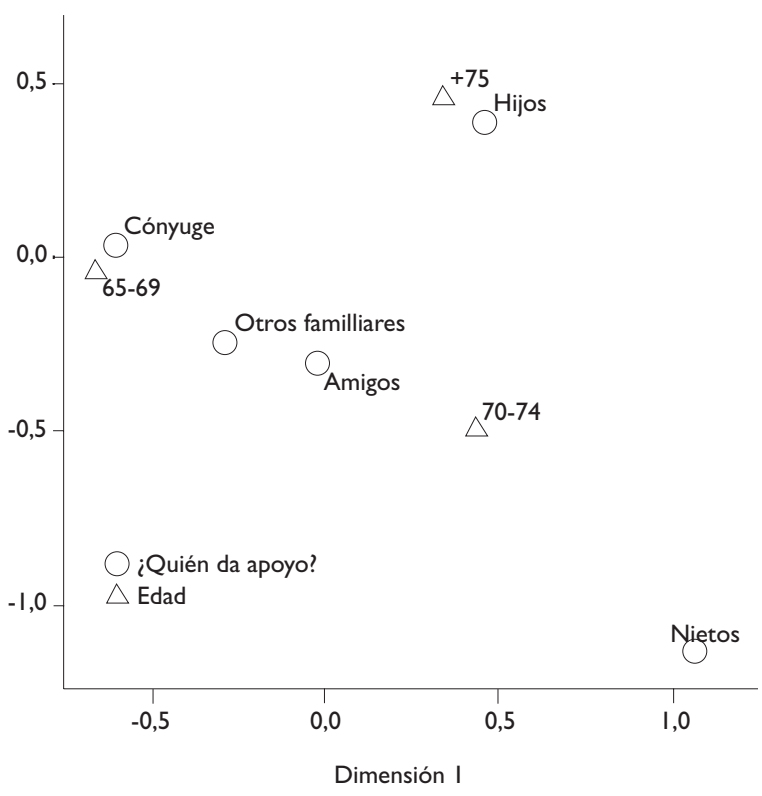

Nota: Entrevista

Figura I. Diagrama dual del anÁlisis de corresponDENCIAS QUE CRUZA LA EDAD CON LA FIGURA DE APOYO PSICOLÓgICo.VALENCIA, EsPaña, ENERO-MAYO 2006

mensión se encontraban el cónyuge, los amigos y otros familiares, con contribuciones respectivas de $-0.53,-0.6$ y -0.63 , mientras en el otro polo estaban los hijos (0.46) y los nietos (0.99). La segunda dimensión fue de muy poca importancia, ya que acumuló tan sólo 3.2\% de la inercia, pero se definió por la oposición entre los entrevistados de 70-74 años (-0.34) y los de 75 o más (0.21), que respectivamente se encontraban vinculados en esta dimensión con otros familiares (-0.40) y amigos (0.41).

\section{Discusión}

Con respecto a la identificación de las personas que suministran apoyo, tal y como se planteaba en la introducción, se ha advertido que, aunque con diferencias en función del tipo de apoyo, es la familia cercana (cónyuge e hijos) la que mayor porcentaje obtiene. Por lo tanto, en el caso del apoyo psicológico, las figuras del cónyuge y los hijos implican $66.7 \%$ en el caso de los hombres y $64.3 \%$ en el de las mujeres; en el apoyo instrumental para los hombres supone $68.3 \%$ y para las mujeres 79.2 por ciento. Estos datos, además de corroborar los ya indicados, ${ }^{9-11}$ reafirman el denominado modelo de con-

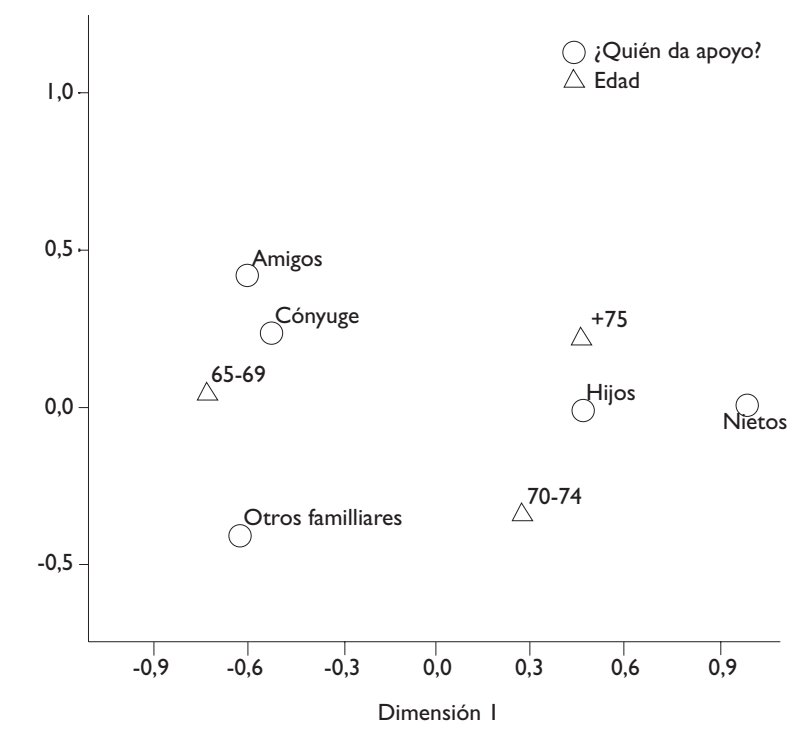

Nota: Entrevista

Figura 2. Diagrama duAL del ANÁlisis DE CORRESPONDENCIAS QUE CRUZA LA EDAD CON LA FIGURA DE APOYO INSTRUMENTAL.VALENCIA, EsPaña, enero-MAYo 2006

voy. ${ }^{20}$ Bajo este modelo se plantea que en los diferentes elementos de cambio que ocurren a lo largo del ciclo vital está presente un número reducido de personas que son las proveedoras del apoyo psicológico e instrumental y que la familia cercana es además la figura más importante en la provisión de este apoyo.

En cuanto al funcionamiento de los tipos de apoyo, según el género, se ha reconocido que, en lo referente al apoyo psicológico, la pareja es para los hombres la figura más importante, seguida de los hijos. En cambio, en el caso de las mujeres, se identifica una clara orientación hacia los hijos como proveedores de apoyo. Para ambos casos, los amigos se convertían en la tercera figura de apoyo, toda vez que éstos son una fuente de refuerzo que se genera a partir de relaciones que son mutuamente provechosas y además contribuyen a favorecer la integración social de las personas mayores.

El matrimonio es más beneficioso para los hombres que para las mujeres, ya que la mayor parte de los esfuerzos para el mantenimiento de las relaciones familiares y del hogar recae en la mujeres; además, estar casado, en el caso de los hombres, significa mayor esperanza de vida, más satisfacción vital y mejor estado de salud. ${ }^{21}$ Por 
otra parte, tanto el posible alejamiento de las relaciones familiares debido al mundo laboral como la pérdida de las relaciones sociales provenientes del trabajo generan una nueva necesidad de acercamiento hacia el cónyuge. En cambio, en el caso de las mujeres, ese esfuerzo por mantener lazos familiares durante las etapas anteriores al envejecimiento hace que se refuercen y mantengan de una forma más clara.

En lo referente al apoyo instrumental, tal y como se ha podido observar, son en ambos casos los hijos la principal figura proveedora de apoyo. De este modo, parece ser que existe una norma de reciprocidad según la cual los hijos ayudan, atienden y cuidan a sus padres de la misma forma que éstos lo hicieron; se produce pues una inversión de funciones que ayuda a minimizar los efectos ejecutivos de la edad para algunas tareas.

Respecto de los datos obtenidos en la relación entre la edad y la figura que ofrece apoyo psicológico, parece relevante subrayar una clara tendencia de cambio a medida que aumenta la edad de los entrevistados. Tal y como se indicaba, la dimensión principal se definió por la oposición entre los más jóvenes y mayores y por el cónyuge frente a hijos y nietos. De este modo, cuando comienza el proceso de la jubilación, el cónyuge se convierte al parecer en la persona más relevante en la superación de situaciones y en la mejoría de la integración; conforme pasan los años, son sobre todo los hijos, y en menor grado los nietos, los que ocupan una posición mucho más relevante. Es posible que el efecto de la viudez influya en este resultado; en cualquier caso, la existencia y acercamiento de los hijos ayudan a generar bienestar en la persona mayor y favorecen la sensación de apoyo psicológico a medida que la edad progresa.

En lo referente al apoyo instrumental, el proceso de cambio con la edad también se ha producido, en apego a la misma tendencia observada en el apoyo psicológico. Es decir, el peso del cónyuge decrece con la edad para dar paso a los hijos y nietos. Parece claro que el nivel de dependencia al realizar actividades cotidianas requiere figuras capaces de dar respuesta a las necesidades de los mayores. Si bien en los primeros momentos éstos pueden apoyarse en su pareja y otros familiares y amigos, y obtener respuestas eficaces, cada vez requieren más el apoyo de los hijos y, en cierto modo, de los nietos, para las actividades instrumentales. Por otra parte, y al igual que en el caso anterior, aunque el efecto de la mortalidad puede producir una reducción del apoyo por parte de los iguales y una mayor tendencia hacia los hijos, el efecto debe ser menor ya que los iguales mantienen características y circunstancias parecidas que les impiden resolver algunas necesidades de los mayores.
Por último, es pertinente a referirse a algunos aspectos. En primer lugar, y en relación con las técnicas estadísticas aplicadas, se ha observado cómo el análisis factorial de correspondencias simple aporta un método gráfico y visual de evaluación de distancia que apoya los resultados de relación de ji cuadrada.

Por otra parte, y en cuanto a la entrevista Manheim de apoyo social, debe señalarse que posee importantes ventajas para el estudio de las redes de apoyo en la vejez y es en particular útil al ofrecer diferentes niveles de análisis: figuras de apoyo (utilizado en este trabajo), frecuencia del contacto, tamaño del apoyo, así como la actitud del entrevistado hacia la búsqueda y recepción del apoyo. Sin embargo, como cualquier entrevista, implica mayor tiempo de aplicación, lo que en la población anciana no siempre es adecuado. En este sentido, la creación de instrumentos más breves para la medida del apoyo tanto funcional como estructural, que sean válidos y adaptados a la población mayor se plantea como un reto dentro del campo de la investigación del envejecimiento.

\section{Referencias}

I. Lin N, Ensel W. Life stress and health: stressors and resources. Am Sociol Rev 1989;54(3):382-399.

2. Gracía E, Herrero J, Musitu G. El apoyo social. Barcelona: PPU, 1995. 3. Lafuente MJ. Hacia una conceptualización del apego. Rev Psicol Educ 1989; | (2):45-55.

4. Bowlby J.Attachment and loss. Vol. I: attachment. Nueva York: Basic Books; 1969.

5. Cantero MJ. Desarrollo socioafectivo. En: Córdoba A, Descals A, Gil MD, eds. Psicología del desarrollo en la edad escolar. Madrid: Pirámide; 2006:157-180.

6. Kahn RL.Aging and social support. In: Riley M, ed. Aging from birth to death. Boulder:Westview; 1979:75-93.

7. Brandtstädter J, Renner G. Tenacious goal pursuit and flexible goal adjustment: explication and age-related analysis of assimilative and accomodative strategies of coping. Psychol Aging 1990;5:58-67. 8. Triadó MC. Envejecer en entornos rurales. Madrid, IMSERSO, Estudios I+D+I, n 19, 2003. [Fecha de publicación: I3/06/2005].

9. INSERSO. Las personas mayores en España. Perfiles. Reciprocidad familiar. Madrid: Instituto Nacional de Servicios Sociales, 1995.

10. Montes de Oca V. Diferencias de género en el sistema de apoyo a la población envejecida en México. Papeles Pobl 1999;19:149-172.

II. Encuesta Nacional sobre Salud y Envejecimiento en México. 200 I. México. Disponible en: http://www.mhas.pop.upenn.edu.

12. Salgado de Snyder VN, González-Vázquez TT, Jáuregui-Ortiz B, BonillaFernández P."No hacen viejos los años, sino los daños": envejecimiento y salud en varones rurales. Salud Publica Mex 2005;47:294-302. 13. Meléndez JC.Apoyo social, tercera edad y autopercepción. Revista de la Sociedad Española de Enfermería Geriátrica y Gerontológica. Gerokomos 1998;9(2):61-67.

14. Schulz R, Heckhausen J. Emotion and control: a life-span perspective. In: Schaie KW, Lawton MP, eds. Annual review of gerontology and geriatrics: emotion and adult development, vol. I7. New York: Springer, 1998. 
15. Vellas P. Envejecer exitosamente: concebir el proceso de envejecimiento con una perspectiva más positiva. Salud Publica Mex 1996;38:5।3-522.

16.Vázquez AJ, García-Bóveda RJ.The Mannheim Interview on Social Support psychometric characteristics of a Spain version. Social Psy Psych Epidemiol 1997;32:208-214

17.Veiel HO.The Mannheim Interview on Social Support. Reliability and validity data from three samples. Social Psy Psych Epidemiol 1990;25(5):250-252.

18. Badia X, Salamero M,Alonso J. Medidas de la salud. Guía de escalas de medición en español. Barcelona: Fundación Lilly, 2002.
19. Abad J, Muñiz N, Cervantes M. Análisis de correspondencias simples y múltiples. En: Lévy JP,Varela J, eds. Análisis multivariable para las ciencias sociales. Madrid: Pearson, Prentice Hall; 2003:36I-4I6.

20. Kahn R,Antonucci TC. Convoys over the life course: attachment, roles and social support life-span development and behaviour (vol. 3). Orlando: Academic Press; 1980.

21. Pinazo S. Relaciones sociales. En:Triadó C,Villar F, coords. Psicología de la vejez. Madrid:Alianza Editorial; 2006:253-285. 